\title{
Téoros
}

Revue de recherche en tourisme

\section{Quoi de neuf chez les routards ?}

\section{Luc Vacher, Maïté Boullosa-Joly et Suzanne Lallemand}

Volume 32, numéro 1, 2013

Tourisme des routards

URI : https://id.erudit.org/iderudit/1036655ar

DOI : https://doi.org/10.7202/1036655ar

Aller au sommaire du numéro

Éditeur(s)

Université du Québec à Montréal

ISSN

0712-8657 (imprimé)

1923-2705 (numérique)

Découvrir la revue

Citer ce document

Vacher, L., Boullosa-Joly, M. \& Lallemand, S. (2013). Quoi de neuf chez les routards ? Téoros, 32(1), 69-72. https://doi.org/10.7202/1036655ar d'utilisation que vous pouvez consulter en ligne.

https://apropos.erudit.org/fr/usagers/politique-dutilisation/ 


\section{Quoi de neuf chez les routards?}

Luc VACHER, Ph.D.

Maître de conférences en géographie

Unité mixte de recherche Littoral environnement

et sociétés (LIENSs)

Université de La Rochelle (France)

Ivacher@univ-Ir.fr
Maïté BOULLOSA-JOLY, Ph.D.

Maître de conférences en anthropologie sociale Centre universitaire de recherches sur l'action publique et le politique (CURAPP)

Université de Picardie Jules Verne (France)

maite.boullosa@wanadoo.fr

Suzanne LALLEMAND, Ph.D.

Docteure en ethnologie

Centre national de la recherche scientifique (France)

suzanne.lallemand@live.fr
La population dont il est question dans ce numéro n'existe pas depuis longtemps ou, plutôt, a été identifiée et dénommée assez tardivement. D'abord, on ne l'a pas dissociée des autres types de voyageurs. Mais, on lui a trouvé des ancêtres : les adolescents anglais, fils d'aristocrates et de gens aisés qui, au XIXe siècle, accomplissent le "Grand tour ", un périple parachevant leur éducation par des visites en Europe du centre et du Sud (Brodsky-Porges, 1981). Puis, des aïeuls : en Amérique, Kerouac et son groupe de jeunes artistes, emblématiques du mouvement de la génération dite perdue, circulent sans relâche sur leur continent dans les années 1940-1950. Ils sont relayés par les fils et petits-fils d'émigrants, moins voyants, étudiants américains qui ont, en fin d'études, envie de connaître l'Europe et le pays dont leur famille est issue. Les Australiens d'origine britannique suivent la même tendance et semblent être les premiers à utiliser le terme «backpackers» (Vacher, 2010), qu'on peut traduire en français par « routards».

Arrivons à la période 1965-1970, celle des grands départs juvéniles, fruits d'un pétrole abondant et de moyens de transport de moins en moins coûteux, mais aussi de la création d'une contre-culture américano-européenne. Celle-ci rejette le monde industriel et vilipende la société de consommation. Pacifiste, elle désapprouve les guerres du moment. Les jeunes itinérants, pour la plupart, approuvent et propagent ces idées. Ils veulent connaître d'autres modes de vie et s'intéressent à d'autres formes de pensée religieuse ou philosophique (Rozenberg 1990; Cohen, 1984). Ils proclament leur refus des travaux routiniers et leur mépris pour la propriété privée. Leur éloignement du sol natal les conduit à une minorisation des liens familiaux, ainsi qu'à une valorisation de ceux acquis lors des déplacements : amitiés immédiates, passions brèves (Riley, 1988), amorces ou longévité de vie en communautés, unions sans mariage. Ils s'annexent des plaisirs nouveaux les célèbres rencontres musicales (Hetherington, 2000) - et s'adonnent à ceux qui leur sont défendus, comme le haschich, l'opium, les champignons hallucinogènes et le LSD.
Lorsqu'ils prennent la route, s'ils admirent une foule de paysages et de monuments et ne dédaignent pas les bords de mer, ils ont aussi des projets de destinations plus stables, lieux où ils s'assemblent volontiers, et que Westerhausen (2002) nomme leurs «sanctuaires» : Ibiza, Goa, Katmandou. Certains d'entre eux y séjournent des années (Rozenberg, 1990). Même si, une fois désargentés, la plupart de ces itinérants finissent par rentrer chez les leurs, ils n’avaient pas fixé la date précise de ce retour.

Dans l'ensemble, les autorités locales des pays en voie de développement où ils se rendent les accueillent mal et les dénigrent : elles leur reprochent d'être sales, drogués, libidineux, paresseux et pauvres. De fait, cette population de voyageurs tranche avec les précédentes. Le voyageur occidental est souvent perçu comme arrogant, très soucieux de son hygiène et, surtout, riche; les jeunes arrivants ne le sont pas et fuient visiblement les grands hôtels destinés aux touristes. Mais, si les douaniers, les policiers et certains journalistes locaux maltraitent ces nouveaux itinérants, une population plus humble leur offre le gîte et le couvert. À la fin de cette période, les chambres chez l'habitant sont saturées. On voit alors se bâtir en grand nombre, du fait d'une petite bourgeoisie active et polyglotte, des maisonnettes électivement destinées à cette clientèle très particulière de jeunes étrangers voyageant sac au dos.

Parfois, d'anciens routards qui se sédentarisent peuvent, pour subvenir à leurs besoins, proposer à leur tour un hébergement à ces jeunes voyageurs occidentaux. Ainsi, en Australie, en Nouvelle-Zélande ou aux États-Unis, des «backpackers hostels» (auberges pour routards) apparaissent, contestant l'ordre et la morale régnant dans le réseau des auberges de jeunesse et autres YMCA (Young Men's Christian Association). Les premières structures peuvent parfois être à la limite de l'autogestion ou du squat.

Que reste-t-il de cette période dans les années 1980 à 2000 ? Le concept du voyage a changé d'ampleur spatiale et de temporalité. Avant la mondialisation, le routard voyageait 
selon un parcours donné et pouvait s'interroger sur le temps qu'il allait passer à chaque endroit. Cette pratique est partiellement révolue : le routard part avec un billet d'avion allerretour, souvent nanti d'une dizaine d'escales, dont les dates de départ et d'arrivée peuvent être soigneusement définies. En revanche, la longueur des déplacements aériens augmente. Le ou les quelques pays visités par leurs prédécesseurs font place au tour du monde, complet ou partiel, surtout chez les plus jeunes voyageurs, qui alignent en peu de mois une fabuleuse liste de pays dans lesquels ils séjournent.

Dans les années 1990, l'intérêt pour la culture des populations d'accueil se refroidit; en Asie de l'Est, peu de routards disent s'être rendus dans un monastère tibétain ou un ashram indien. Les musées locaux les intéressent médiocrement et ils ne se satisfont guère des visites de monuments, trop fréquentés à leur goût par les touristes. En revanche, le sport apparait comme leur principale activité. À la recherche d'émotions fortes et de vertige, ils pratiquent la nage, le surf, le rafting, le saut à l'élastique et la plongée sous-marine (surtout avec tuba). Ils escaladent montagnes et volcans, s'enfoncent dans la jungle des jours durant, marchent vers des villages où ne mène aucune route carrossable. La curiosité des routards se porte aussi vers les sports locaux (lutte, boxe thaïe) et les arts de la danse, ainsi que vers les thérapies du massage, vis-à-vis desquelles ils sont volontiers clients et apprentis potentiels. L'attention portée aux techniques du corps tend à se substituer largement aux aspirations philosophico-religieuses d'une période déjà lointaine.

Sur le plan des gites, l'invitation chez l'habitant est un souvenir que quelques adroits solitaires savent néanmoins ressusciter. Désormais, de nombreux hôtels attendent les jeunes voyageurs étrangers. Pour les habitants locaux de leur âge, ils sont trop chers; pour les étrangers d'âge moyen, ils sont inconfortables. Cependant, ils sont parfaits pour les routards qui se retrouvent entre eux, sans paraître remarquer l'aimable ghettoïsation dont ils sont l'objet. Les hôteliers leur proposent des guides, des spectacles et des excursions. Ils préfèrent se livrer longuement à une autre activité : socialiser ensemble. Faire connaissance, se raconter, entendre le récit de vie d'autrui, se quitter, se retrouver mille kilomètres plus loin, constituer un groupe, puis un duo, etc. (Lallemand, 2010). En bref, rencontrer aussi loin que possible des gens qui leur ressemblent et qu'ils connaissent déjà.

En ce qui concerne les relations avec les autorités, signalons qu'elles sont excellentes. Ils ont peu de contact avec les gens de leur âge, qui ne les distinguent pas des autres touristes. Ils sont aussi follement enviés par les jeunes qui les approchent - garçons d'hôtel, guides, serveuses qui rêvent de leur liberté et les imaginent fortunés (ce qu'ils sont dans beaucoup de pays visités, mais pas dans leur pays d'origine). De 1965 à 2000 , les routards semblent donc avoir beaucoup changé. Mais qu'en est-il actuellement?

En fait, il est bien possible qu'au-delà des pratiques de voyage, ce soit le contexte technologique qui ait le plus changé en ce début de $\mathrm{XXI}^{e}$ siècle, en particulier en matière de communication. Le transport aérien est plus rapide et abordable, et les communications ont été révolutionnées par la diffusion de l'Internet jusque dans les villages les plus reculés de certains pays d'Asie du Sud-est ou d'Afrique du Nord. Les réseaux de téléphonie mobile sont particulièrement importants dans les pays du sud, où ils concernent aujourd'hui beaucoup plus de gens que les traditionnels réseaux filaires. Ces technologies adaptées à la gestion du tourisme ont facilité l'expansion de celui-ci, tout comme elles ont permis le développement de nouvelles opportunités pour les jeunes voyageurs. Bien sûr, en conséquence, les notions d'aventure, de prise de risques et de mise à distance ont elles aussi évolué.

Dans les transformations récentes relatives à la mobilité des jeunes voyageurs, nous pouvons noter l'importance croissante des pratiques urbaines, en particulier dans les grandes métropoles, qui apparaissent plus que jamais comme un formidable terrain d'aventure. La pratique touristique de la ville par les jeunes n'est pas nouvelle, mais elle est aujourd'hui simplifiée. On peut distinguer trois ressorts à cette simplification, qui jouent d'ailleurs sur l'ensemble de la cartographie touristique actuelle.

Le premier concerne le fait que le développement des réseaux de télécommunication, en particulier Internet et la téléphonie mobile, a facilité la mise en contact entre le producteur de services touristiques et ses potentiels clients. L'élaboration d'un site Internet pour faire la promotion d'un hébergement dans une vallée perdue du Maroc est aujourd'hui à la portée de bon nombre de petits porteurs de projets, éventuellement avec l'aide de jeunes visiteurs. L'interaction possible entre des acteurs locaux et les jeunes touristes très connectés se développe ainsi, au fur et à mesure de l'amélioration du réseau à l'échelle mondiale. Ces rapports, souvent épaulés par les forums et blogues de voyageurs, permettent de se dispenser des intermédiaires, de leurs profits et de leurs logiques de concentration de l'offre. Ils remplacent ou complètent la diffusion de l'information par le bouche-à-oreille ou les guides papier, qui ont longtemps été les modes dominants de diffusion de l'information chez les routards.

Un deuxième ressort fondamental de l'évolution des pratiques est là encore lié au développement des nouvelles technologies de communication : l'immédiateté. Il est aujourd'hui classique pour un jeune voyageur de réserver ou d'acheter un billet d'avion ou un hébergement en ligne, et cela au cours de son voyage. Cet achat, qui l'autorise à ne pas tout prévoir avant son départ, pourra se faire depuis sa chambre, voire depuis le hall de son auberge, grâce à une connexion Internet par un réseau Wi-Fi. Cet accès immédiat à l'information et au geste d'achat lui permet d'être plus réactif aux sollicitations du lieu et de moins planifier son voyage.

Le troisième ressort, qui découle en partie des deux autres, est l'autonomisation des jeunes voyageurs. L'achat d'un billet d'avion devient plus simple quand on le fait soi-même, au moyen du réseau Wi-Fi de l'auberge, qu'en faisant la queue pour s'en remettre à un agent de voyage, qui respectera les heures d'ouverture et de fermeture d'une agence. Il n'est pas impossible de trouver un hébergement gratuit pour la semaine suivante en Colombie grâce au réseau CouchSurfing. Celui-ci permet également d'entrer en contact avec de jeunes habitants locaux à la recherche de sociabilités, avec qui on pourra découvrir et partager un mode de vie «authentique», comme en rêvent de nombreux jeunes voyageurs. L'autonomisation se 
traduit aussi par la possibilité, pour le voyageur, d'explorer des destinations qui n'auraient pas de rentabilité dans un système d'exploitation commerciale classique. En effet, avec les pratiques par lesquelles il acquiert des compétences (un capital de savoir-faire), il peut gérer de façon autonome les différentes étapes de construction d'un voyage.

Nous verrons comment ces différentes facettes du voyage des routards du début du XXIe siècle peuvent prendre forme à travers les articles qui composent ce numéro.

Les contributions présentées ici montrent, à partir d'enquêtes récentes, les multiples aspects du voyage itinérant contemporain et le rôle des nouvelles technologies dans l'évolution de ces périples, qui n'ont plus toujours pour destination les contrées lointaines pour le monde occidental. Brenda LE BIGOT et Antoine FLEURY décrivent la pratique du couchsurfing à Paris et à Berlin. Ils expliquent comment cette démarche permet de découvrir la ville européenne autrement et sans trop dépenser. Les auteurs ont mené leur enquête en recourant aux mêmes moyens modernes de communication et c'est grâce au site CouchSurfing.org qu'ils ont pu trouver des interlocuteurs pour réaliser leurs entretiens. L'article décrit le déroulement des séjours de ceux qui choisissent le couchsurfing comme moyen d'hébergement et porte d'entrée pour aller à la rencontre de l'autre. On découvre là un mode de mobilité original, où l'hôte joue un rôle déterminant dans la réalisation du périple et a une influence majeure sur la représentation des villes visitées. Leur analyse met en évidence la place croissante d'Internet dans la préparation des voyages, que ce soit pour le mode de transport, le séjour ou l'hébergement. Mais l'Internet permet aussi de mettre en récit le voyage et d'avoir accès aux représentations que les routards se font de leur expérience ou désirent que l'on s'en fasse. Comme le soulignent les auteurs, l'Internet permet de créer une cartographie de sa propre mobilité et ils interrogent ici l'influence du couchsurfing sur les représentations des lieux visités.

Afin d'approfondir les représentations qu'ont les routards de leur périple et le sens qu'ils lui donnent, Julien COURANT décrit en détail les carnets que tiennent, au jour le jour, ces voyageurs au long cours. Nous ne sommes plus dans les nouvelles technologies de communication, mais dans l'écriture du carnet, l'équivalent du journal intime. Julien Courant a côtoyé, depuis dix ans, ces routards afin d'en connaitre certains et d'avoir accès à leurs carnets, souvent écrits pour eux-mêmes ou leurs confidents. Il décrit ces objets qui accompagnent les routards dans leur quotidien et les différentes formes qu'ils peuvent prendre. Julien Courant détaille aussi leur contenu avec l'importance accordée à l'exotisme et à l'imprévu comme éléments significatifs de la capacité d'adaptation des voyageurs aventureux. L'auteur nous amène ainsi à réfléchir sur le sens de cette écriture quotidienne, la manière dont elle est mise en mots et la façon dont s'exprime la dimension initiatique de l'expérience itinérante.

Le rapport au temps de ces aventuriers est analysé par Jocelyn LACHANCE, à partir de la littérature scientifique anglo-saxonne sur le sujet. L'auteur montre la spécificité de ces voyageurs qui, à l'opposé du tourisme de masse, ne suivent pas un rythme imposé par un guide ou un groupe. Cela ne sous-entend pas pour autant qu'il n'y ait pas de leur part une maîtrise du temps, bien au contraire. L'auteur décrit la façon dont les rythmes du voyage sont déterminés par les routards et comment cela participe à singulariser leur parcours. La possibilité d'étirer le temps d'un séjour apparaît d'ailleurs comme un moyen de distinction par rapport au tourisme de masse. L'auteur analyse comment s'opère la rupture provisoire avec les contraintes horaires quotidiennes et la façon dont ces voyageurs créent des rythmes personnels et réorganisent leur temporalité du voyage par le récit. La question de l'influence de l'usage des nouvelles technologies dans ce rapport au temps est aussi posée.

La contribution de Gwendal SIMON porte, pour sa part, sur le débat entourant l'institutionnalisation des pratiques des routards, en décrivant leurs visites d'un lieu hautement touristique : Paris. Il ne s'agit donc pas de périples aventureux des routards loin du monde occidental, mais bien de leur approche des espaces urbains que l'on n'avait pas l'habitude de voir analysée jusque-là. L'auteur dépeint leur découverte de la ville à l'écart des grands circuits touristiques et leur représentation singulière de cette expérience. Pour son enquête, Gwendal Simon a mené des entretiens dans des auberges de jeunesse parisiennes avec des routards de plusieurs nationalités (européennes, nord et sud-américaines, australienne), âgés de 17 à 29 ans et voyageant dans le cadre d'un circuit libre à travers l'Europe. L'attention a été principalement centrée sur les approches diversifiées de la capitale française par les routards et leur façon singulière de vivre cette expérience touristique urbaine souvent stéréotypée sur la scène touristique mondiale. L'auteur montre comment leur démarche est marquée par une autonomie dans les pratiques et la façon dont ils s'y prennent pour vivre des expériences originales non dénaturées par l'industrie du voyage et des imaginaires touristiques trop standardisés.

Nous verrons cependant que cette lutte, de la part des routards, pour se distinguer d'un tourisme devenu conventionnel n'existe pas seulement dans les capitales européennes, mais aussi dans les régions polaires. Antoine DELMAS montre en effet, dans son article sur le Groenland, les incidences de la venue de routards sur les lieux qu'ils visitent et leur participation à la «touristification» (mise en tourisme) de ces espaces. Du routard au croisiériste de luxe, il décrit précisément les différents types de tourisme dans cette région polaire et met en lumière leurs spécificités, mais aussi leurs points communs. Ses sources proviennent de travaux de terrain menés en 2011 et 2012, où il a réalisé de nombreux entretiens avec des acteurs locaux du tourisme et différents types de visiteurs, qui lui ont livré leurs aspirations. Si les routards ont nourri le rêve de cette expédition, les visiteurs plus fortunés semblent davantage dans une démarche consommatrice, où la quête d'aventure a laissé place à un certain confort que les premiers regrettent. Pour en revenir aux nouvelles technologies et à leur incidence sur les transformations du voyage, Antoine Delmas mentionne le site du bureau du tourisme du Groenland, qui permet au visiteur de choisir le degré d'aventure qu'il souhaite vivre lors de son voyage en zone polaire. La touristification des lieux balise ainsi l'expérience et incite les routards à aller voir plus loin, hors des sentiers battus. Les routards ne sont d'ailleurs plus désirés dans les lieux qu'ils ont participé à faire connaître, car ils sont 
mis en concurrence avec un tourisme plus lucratif qui génère d'importantes retombées économiques. C'est ainsi qu'ils cherchent d'autres lieux plus sauvages... Antoine Delmas décrit ce processus comme un inexorable mécanisme qui reprendrait les caractéristiques des fronts pionniers «qui ne trouvent de limites que dans la finitude de l'espace géographique».

Toutes ces contributions nous montrent bien que ce ne sont plus les lieux de destination qui caractérisent aujourd'hui ces voyageurs itinérants, mais plutôt les pratiques de ces derniers, qui font preuve d'une créativité perpétuelle pour continuer à explorer le monde en évitant les sentiers trop coûteux et standardisés.

\section{Références}

BRODSKY-PORGES, Edward (1981) «The Grand Tour Travel as an

Educational Device 1600-1800", Annals of Tourism Research, vol. 8, n² 2, p. 171-186.

COHEN, Eric (1984) «Notes on the Phenomenon of Drifter-Tourism »,

International Journal of Comparative Sociology, vol. 14, $\mathrm{n}^{\circ}$ 1-2, p. 89-103. HETHERINGTON, Kevin (2000) New Age Travellers: Vanloads of Uproarious

Humanity, Londres : Cassell. $191 \mathrm{p}$.
KEROUAC, Jack (1957) On the Road, New York : Viking Press. 320 p.

LALLEMAND, Suzanne (2010) Routards en Asie, ethnologie d'un tourisme voyageur, Paris : L'Harmattan. 295 p.

RILEY, Pamela J. (1988) « Road Culture of International Long-Term Budget Travellers ", Annals of Tourism Research, vol. $15, \mathrm{n}^{\circ}$ 3, p. 313-328.

ROZENBERG, Danielle (1990) Tourisme et utopie aux Baléares, Ibiza, une île pour une autre vie, Paris : L'Harmattan. 200 p.

VACHER, Luc (2010) «Du Grand Tour au tour du monde des backpackers : la dimension initiatique dans le voyage touristique», DANS TISSOT, Laurent (sous la direction de), Attrait d'ailleurs. Images, usages et espaces du voyage à l'époque contemporaine, Paris : Éditions du CTHS. p. 113-122.

WETERHAUSEN, Klaus (2002) Behond the Beach. An Ethnography of Modern Travellers in Asia, Bangkok: White Lotus Press. 272 p.

\section{ANS D'EXPÉRIENCE EN TOURISME Complétez votre collection}

$\square$ Le tout-inclus

vol. $31, n^{0} 2,2012$

$\square$ Gouvernance des parcs au Nunavik vol. $31, n^{0} 1,2012$

$\square$ Tourisme et patrimoine mondial Les associations de patrimoine et le tourisme vol. $30, n^{\circ} 2,2011$

$\square$ Excellence des destinations Ciné-tourisme vol. $30, n^{0} 1,2011$

$\square$ Géotourisme / Tourisme et femmes vol. 29, $n^{\circ} 2,2010$

$\square$ Tourisme des racines Tourisme et autochtones vol. $29, n^{0} 1,2010$

$\square$ Tourisme, sport et développement vol. 28, no 2, 2009

$\square$ Tourisme polaire vol. 28, no 1, 2009 $\square$ Nouveaux musées, nouveaux tourismes vol. $27, n^{\circ} 3$, automne 2008

$\square$ Les grands équipements touristiques vol. $27, n^{\circ} 2$, été 2008

$\square$ La science du tourisme vol. $27, n^{0} 1$, printemps 2008

$\square$ Tourisme et solidarité vol. $26, n^{\circ} 3$, automne 2007

$\square$ Tourisme et attractivité vol. $26, n^{\circ} 2$, été 2007

$\square$ Tourisme Caraïbe vol. $26, n^{0} 1$, printemps 2007

$\square$ Forêt vol. $25, n^{\circ} 3$, automne 2006

$\square$ Désirs d'Orient vol. 25, nº 2, été 2006

$\square$ Tourisme gourmand vol. $25, n^{0} 1$, printemps 2006 $\square$ Tourisme et santé vol. 24, no 3, automne 2005

$\square$ Tourisme, religion et patrimoine vol. 24, n² 2, été 2005

$\square$ Maroc vol. $24, n^{0} 1$, printemps 2005

$\square$ Regards sur l'hôtellerie vol. $23, n^{0} 3$, automne 2004

$\square$ La qualité en tourisme vol. $23, n^{\circ} 2$, été 2004

$\square$ Au risque du politique vol. $23, n^{0} 1$, printemps 2004

$\square$ L'accessibilité, une conquête inachevée vol. 22, nº 3, automne 2003

$\square$ Les routes à thèmes vol. 22, n² 2, été 2003

$\square$ Tourisme et sexualité vol. 22, $n^{0} 1$, printemps 2003

$20 \$$ / numéro, taxes incluses. Livraison gratuite pour tout achat de plus de 50 . Faites vos achats sur www.coopugam.com. 